\title{
Level of inflammatory cytokines tumour necrosis factor $\alpha$, interleukins 12, 23 and 17 in patients with psoriasis in the context of metabolic syndrome
}

\author{
Magdalena Pirowska, Katarzyna Podolec, Sylwia Lipko-Godlewska, Joanna Sułowicz, Paweł Brzewski, \\ Aleksander Obtułowicz, Maciej Pastuszczak, Anna Wojas-Pelc
}

Department of Dermatology, Jagiellonian University Medical College, Krakow, Poland

Adv Dermatol Allergol 2019; XXXVI (1): 70-75 DOI: https://doi.org/10.5114/ada.2018.73136

\begin{abstract}
Introduction: Psoriasis is a chronic inflammatory skin disease with immunologic etiology.

Aim: To investigate the levels of the proinflammatory cytokines tumor necrosis factor $\alpha$ (TNF- $\alpha$ ), interleukin 23 (IL-23) and IL-17 in patients with psoriasis and psoriatic arthritis with concomitant metabolic syndrome.

Material and methods: This study included 60 patients with severe psoriasis.

Results: In patients with arterial hypertension concomitant with psoriasis, no statistically significant differences in cytokine levels were observed. On the other hand, in the group of patients diagnosed with diabetes, an increased level of IL-17 was observed. In patients with lipid disorders, the results were similar to the results of patients with diabetes.

Conclusions: It is very important to study immunologic mechanisms responsible for the presence and severity of psoriasis, in order to personalize the therapy in the future and optimize the effect of action on the basic disease and on concomitant disorders.
\end{abstract}

Key words: psoriasis, metabolic syndrome, cytokines.

\section{Introduction}

Psoriasis is a chronic inflammatory skin disease with immunologic etiology, depending on the activation of Th-1, Th-17 and Th-22 lymphocytes and dendritic cells [1]. In recent years, scientific research has been focusing on the identification of inflammatory pathways engaged in the pathogenesis of psoriasis. Several inflammatory cytokines are produced in skin lesions (TNF, IL-1, IL-6, IL-8, IL-17, IL-22, IL-23, VEGF, IFN- $\gamma$ ) [2]. It seems that these molecules are released into the circulatory system in a concentration depending on the severity and extent of the lesions [3, 4]. Furthermore, experimental and epidemiologic studies indicate an association of increased levels of interleukins, cytokines and hormones (adipokine) with cardiovascular diseases (CVD), metabolic syndrome (MetS), obesity and diabetes, defining psoriasis as a risk factor for the development of systemic diseases [2, 5, 6]. Apart from chronic inflammation of skin, also other common risk factors should be considered (smoking, drinking alcohol), treatment used (immunosuppressive and changing the lipid profile), as well as common loci of predisposing genes [7].

Danielsen et al. proved that concomitant psoriasis is related to 3.8-fold higher risk of developing metabolic syndrome in women aged 30 and 1.35-fold higher in men, independent of age [8]. Langan et al. [9] suggest performing screening tests for the features of metabolic syndrome in patients with severe psoriasis, as the risk of their occurrence significantly increases with the increase of the severity of skin lesions. Azfar et al. [10] report significantly increased risk of occurrence of type 2 diabetes in patients with severe psoriasis $(H R=1.46)$. Ni et al. [5] found that the frequency of severe vascular episodes (infarction, stroke, cardiovascular death) in patients with psoriasis is significantly higher than in the group without dermatosis (4.9\% vs. 2.9\%). These findings are also confirmed in the study by Armstrong et al. [11], giving an increase of the risk of infarction $(R R=1.29)$, stroke $(R R=$ $1.12)$ and death for cardiovascular reasons $(R R=1.39)$ in patients with psoriasis.

Address for correspondence: Magdalena Pirowska PhD, Department of Dermatology, Jagiellonian University Medical College, 8 Skawinska St, 30-066 Krakow, Poland, phone: +48 600858 891, e-mail: pirowska@gmail.com

Received: 3.10.2017, accepted: 1.12.2017. 
It is still an open question whether we can reduce the risk of the above incidents by appropriate treatment of skin lesions, in consequence reducing generalized inflammation. Numerous studies [12-14] report that treatment with methotrexate (MTX) reduces the risk of developing CVD by $21-27 \%$ and the risk of myocardial infarction by $18 \%$. The mechanism of action remains unclear; MTX has been shown to downregulate foam cell production and increase expression of anti-atherogenic reverse cholesteroltransport protein [14]. To date, the positive influence of biopharmaceuticals has not been unambiguously confirmed. The National Psoriasis Foundation reports that anti-TNF drugs reduce the risk of cardiovascular episodes [15]. Most data concerning the use of ustekinumab are relatively short-term, but they suggest no influence of the drug on CVD. There are also single reports indicating reduced risk [11]. A meta-analysis of 34 studies concerning patients treated for psoriasis and psoriatic arthritis confirmed that methotrexate and anti-TNF reduce the risk of CVD in the absence of a protective effect of ciclosporin A, retinoids and anti-IL-12/23 [11, 16].

\section{Aim}

The aim of the study was to investigate the levels of proinflammatory cytokines tumor necrosis factor $\alpha$ (TNF- $\alpha$ ), interleukin 23 (IL-12), IL-23 and IL-17 in patients with psoriasis and psoriatic arthritis with concomitant metabolic syndrome in the context of individual components of this syndrome (abdominal obesity, diabetes, arterial hypertension, hyperlipidemia). Linking an elevated level of a specific interleukin with the presence of specific disorders in the course of psoriasis could influence therapeutic decisions and lead to personalization of therapy. Individual biopharmaceuticals against cytokines could additionally improve the parameters of metabolic diseases and help to manage diabetes or hyperlipidemia.

\section{Material and methods}

This study included 60 patients over 18 years of age (24 female and 36 male), diagnosed and treated for a severe psoriasis (Psoriasis Area Severity Index (PASI), body surface area $(B S A)>10)$ in a dermatology clinic. Control groups were: 15 subjects diagnosed with metabolic syndrome and 15 healthy voluntary subjects. In both groups, the exclusion criteria were acute and chronic inflammatory diseases with the exception of diabetes, hypertension and dyslipidemia.

For testing, venous blood $(5 \mathrm{ml})$ was collected after the night rest. The concentrations of subunit p70 IL-12, IL-17 and IL-23 and TNF- $\alpha$ in subjects' sera were determined by the Quantikine test with enhanced sensitivity, with the ELISA method. The expected values were: IL-12 ND (below) - $3.58 \mathrm{pg} / \mathrm{ml}$; IL-17 below $31.2 \mathrm{pg} / \mathrm{ml}$; IL-23 ND (below) $-40.5 \mathrm{pg} / \mathrm{ml}$; TNF- $\alpha 0.550-2.816 \mathrm{pg} / \mathrm{ml}$. The severity of psoriasis was evaluated with PASI and BSA scales. Psoriatic arthritis was diagnosed based on the Caspar criteria. Metabolic syndrome was diagnosed based on the presence of revised National Cholesterol Education Program - Adult Treatment Panel III (NCEP-ATP III) criteria for metabolic syndrome, according to American Heart Association/ The National Heart, Lung and Blood Institute (ANA/NHBLI).

For conducting the above studies, the consent of the Bioethics Committee of Jagiellonian University was obtained.

\section{Statistical analysis}

For the statistical assessment, the Statistica program was used (basic statistics, t-test, Mann-Whitney U-test, Spearman correlation).

\section{Results}

In studied subjects, the average age was $49.4 \pm 16.4$ years; the youngest patient was 20 , the oldest one was 82 . The IL-12 level in all the subjects was indeterminable
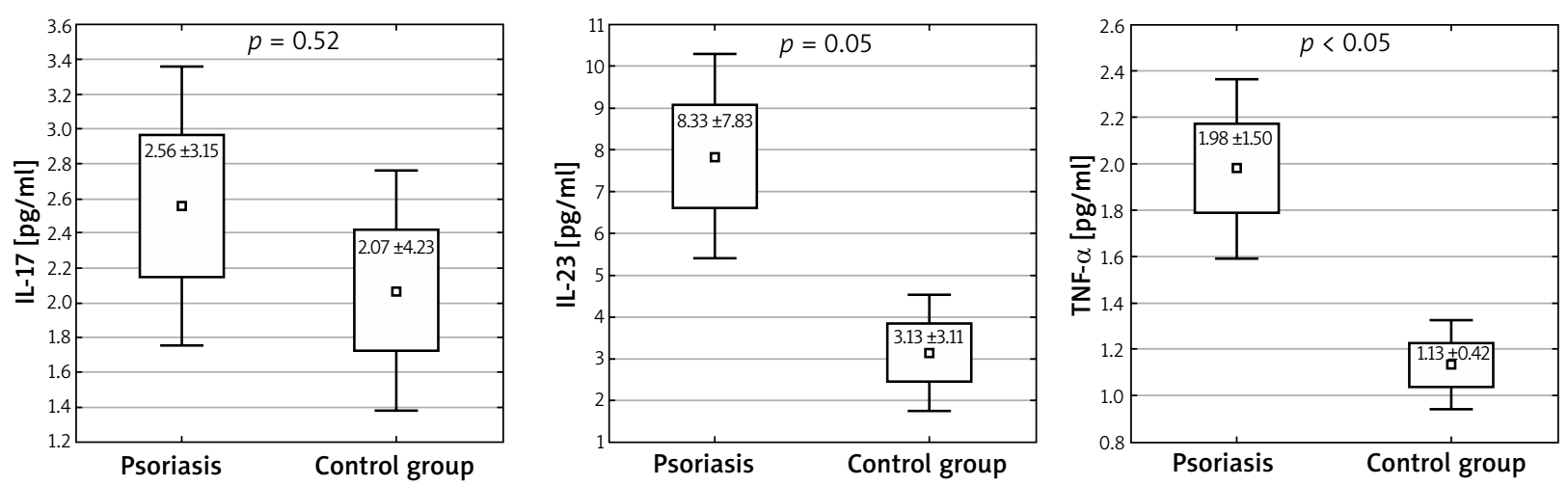

Figure 1. Serum concentrations of IL-17, IL-23 and TNF- $\alpha$ in the study and control group. Mann-Whitney $U$ test was performed to evaluate differences between the control and study group (mean $\pm \mathrm{SD}, \pm 1.96^{*} \mathrm{SD}$ ) 

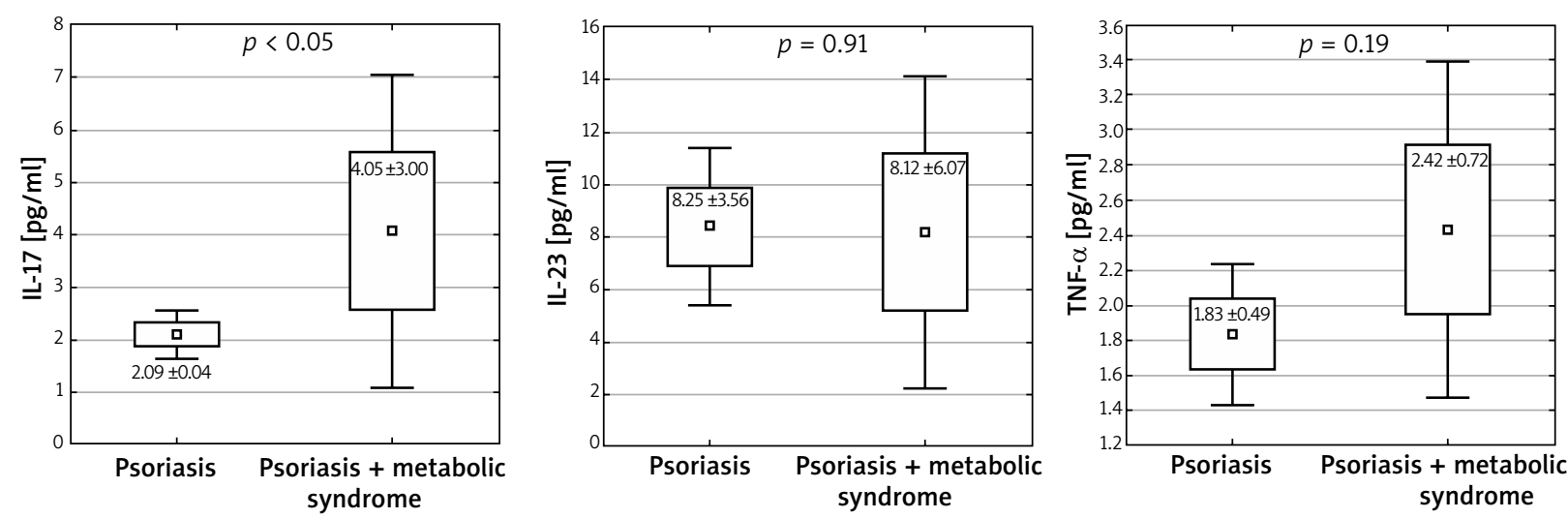

Figure 2. Levels of cytokines in patients with metabolic syndrome accompanying psoriasis in comparison to patients with a chronic dermatosis without the features of this syndrome (mean $\pm \mathrm{SD}, \pm 1.96^{\star} \mathrm{SD}$ )
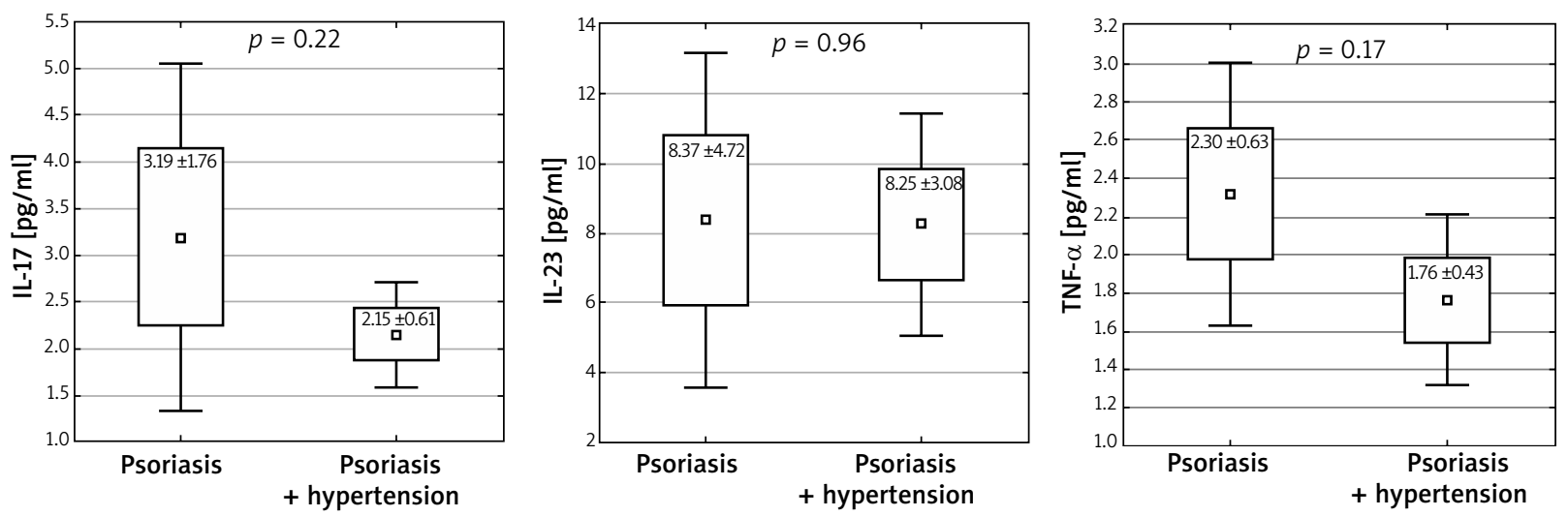

Figure 3. Levels of studied cytokines in patients with arterial hypertension concomitant with psoriasis (mean \pm SD, $\pm 1.96^{\star} \mathrm{SD}$ )
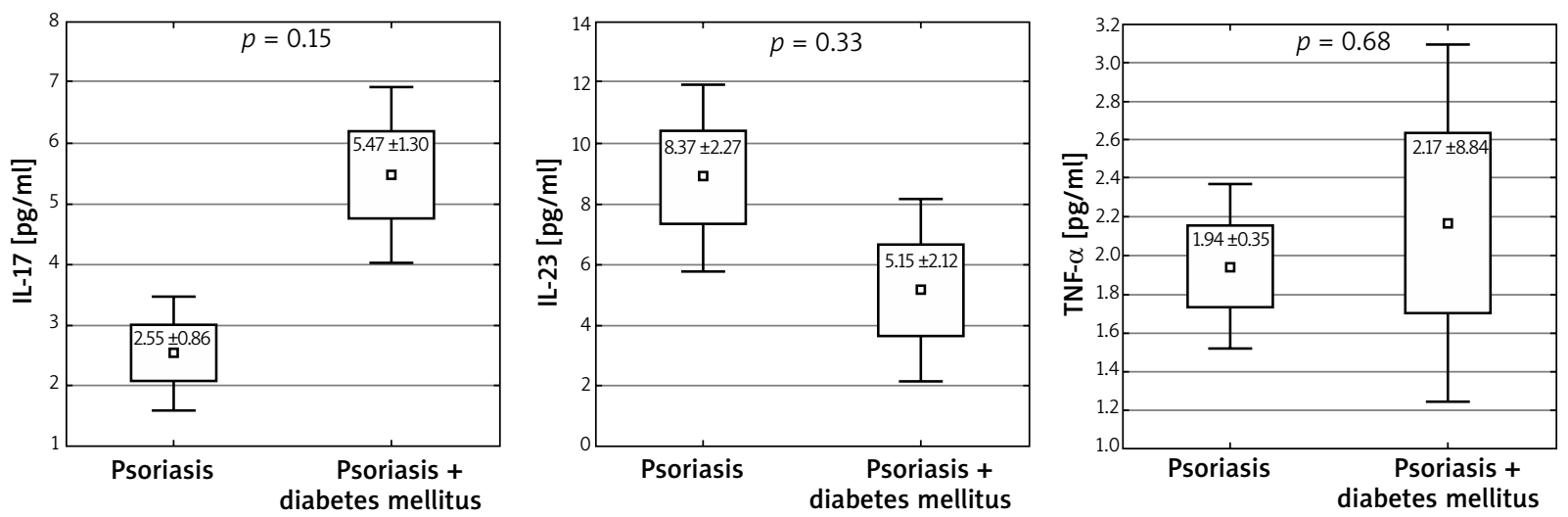

Figure 4. Levels of studied cytokines in patients with diabetes mellitus concomitant with psoriasis (mean \pm SD, $\pm 1.96^{\star} S D$ ) 

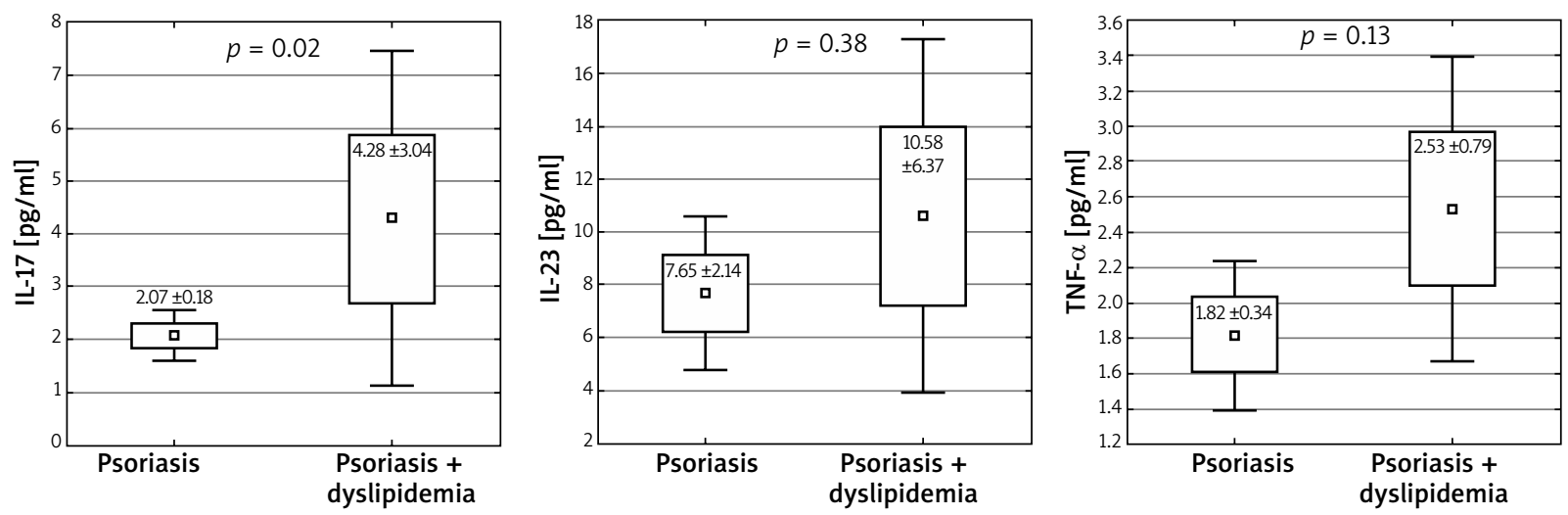

Figure 5 . Levels of studied cytokines in patients with dyslipidemia concomitant with psoriasis (mean $\pm S D, \pm 1.96^{\star} S D$ )

$(<0.78 \mathrm{pg} / \mathrm{ml})$. The level of the studied cytokines IL-23 and TNF- $\alpha$ was higher in patients diagnosed with psoriasis than in the control group: IL-23: $8.33 \pm 7.83 \mathrm{pg} / \mathrm{ml}$ vs. $3.13 \pm 3.11 \mathrm{pg} / \mathrm{ml} ; p<0.05,1-\beta$ (false negative) = 0.998; TNF- $\alpha: 1.98 \pm 1.50 \mathrm{pg} / \mathrm{ml}$ vs. $1.13 \pm 0.42 \mathrm{pg} / \mathrm{ml} ; p<0.05$, $1-\beta=0.993$ (Figure 1). Higher levels of IL-17 were observed in patients with metabolic syndrome accompanying psoriasis than in patients with a chronic dermatosis without the features of this syndrome: IL-17: 2.09 $\pm 0.04 \mathrm{pg} / \mathrm{ml}$ vs. $4.06 \pm 3.00 \mathrm{pg} / \mathrm{ml} ; p<0.05,1-\beta=0.973$ (Figure 2). Among the patients with psoriasis and psoriatic arthritis, no correlation was observed between the level of cytokines and BMI. There were no correlations between the waist circumference and investigated cytokines.

In patients with arterial hypertension concomitant with psoriasis, no statistically significant differences in interleukin levels were observed (IL-17: 2.15 vs. 3.19 pg/ $\mathrm{ml}, p=0.22$; IL-23: 8.25 vs. $8.37 \mathrm{pg} / \mathrm{ml}, p=0.96$; TNF- $\alpha$ : 1.76 vs. $2.3 \mathrm{pg} / \mathrm{ml}, p=0.17,1-\beta=0.693$ (Figure 3). On the other hand, in the group of patients diagnosed with diabetes, an increased level of IL-17 was observed (2.55 vs. $5.4 \mathrm{pg} / \mathrm{ml}, p=0.015$ ) (Figure 4).

In patients with lipid disorders, the results were similar to the results of patients with diabetes. An increased level of IL-17 was observed (4.28 vs. $2.07 \mathrm{pg} / \mathrm{ml}, p=0.02$; $1-\beta=0.776)$, while for the levels of other interleukins the obtained differences were statistically insignificant (IL-23: 10.58 vs. $7.65 \mathrm{pg} / \mathrm{ml} ; p=0.38$ and TNF- $\alpha$ : 2.53 vs. $1.82 \mathrm{pg} / \mathrm{ml} ; p=0.13$ ) (Figure 5).

\section{Discussion}

The incidence of type 2 diabetes is significantly increased in patients with psoriasis. Azfar et al. [10] claim that the presence of psoriasis is an independent risk factor for the development of type 2 diabetes. Importantly, Ucak et al. found that insulin resistance occurs with increased frequency in patients with psoriasis who are not obese [17]. Moreover, a positive correlation of insulin resistance with PASI index was proven [18]. Previous studies state that the main mediators responsible for the development of insulin resistance are TNF- $\alpha$, IL-6, leptin, adiponectin, or cytokines playing an important role in etiopathogenesis of psoriasis [19, 20]. Solomon et al. [21] found that the risk of diabetes development decreases in patients treated with TNF- $\alpha$ inhibitors compared to patients taking disease-modifying antirheumatic drugs (DMARD). Other studies also prove a decrease of insulin resistance with anti-TNF- $\alpha$ treatment [22]. Nevertheless, the results of studies on the influence of biopharmaceuticals on diseases constituting components of metabolic syndrome - diabetes, hypertension and dyslipidemia are different.

Wu et al. [23] did not find significant changes as to the values of arterial pressure, blood glucose level and lipid profile in patients with psoriasis treated with TNF- $\alpha$ inhibitors compared to patients taking MTX. On the other hand, Famenini et al. [24] confirm a positive effect of anti-TNF- $\alpha$ therapy on reducing the risk of CVD development; however, the influence of drugs on blood glucose profile is various (there are cases reporting hyper- as well as hypoglycemia after administration). There are no reports concerning the influence of other biopharmaceuticals used in psoriasis treatment (blocking IL-12/23, IL-17) on the development and course of metabolic diseases. Only Eiris et al. [25] found that in the Spanish population, genetic variability in IL-12B, IL-23R and IL-23A affects not only the risk of psoriasis development, but also occurrence and severity of type 2 diabetes. On the other hand, in recent years, the influence of Th17 lymphocytes and the IL-17/IFN- $\gamma$ axis on the development of type 1 diabetes has been described $[26,27]$. In the context of the above-mentioned reports, Roohi et al. [28] assessed the level of IL-17 and IL-23 in patients with type 1 and type 2 diabetes. Our study showed a significantly increased level of IL-17 in patients with type 2 diabetes concomitant with 
psoriasis. No statistically significant differences in levels of IL-23 and TNF- $\alpha$ were found. Considering the described results, it is recommended to extend the study with the assessment of blood glucose levels and insulin resistance in patients treated with IL-17 inhibitors. A confirmation of the effect of the therapy on the course of diabetes and insulin resistance could indirectly significantly reduce the risk of CVD development and death [28].

The authors of a large systematic review covering 2,400,000 patients reported that patients with psoriasis have a higher incidence of dyslipidemia than the general population [29]. Issues concerning the correlation of psoriasis, lipid metabolism and IL-17 were discussed in detail by Owczarczyk-Saczonek et al. [30] Varshney et al. [31] found that IL-17A leads to the accumulation of cholesterol in keratinocytes, which can play a crucial role in pathophysiology of psoriasis. An increased level of IL-17 correlates with a significant increase of intracellular level of cholesterol, which could explain frequent co-morbidity of psoriasis and dyslipidemia. According to our knowledge, no research has been performed directly concerning the level of IL-17 in patients with the described dermatoses and disorders of lipid profile. The results obtained in our study confirm the observations of Varshney et al., indicating the level of IL-17 as important in the treatment of patients with dyslipidemia and psoriasis [31].

The correlation of arterial hypertension with cytokine profile is not so obvious, as the development of hypertension is also affected by the sympathetic system, renal disorders and vascular disorders [30]. The studies conducted among patients with diabetes confirmed a significantly elevated level of IL-17A in subjects with concomitant hypertension compared to subjects with normal blood pressure [32]. It should also be noted that there are reports about the influence of briakinumab (inhibiting $\mathrm{IL}-12 / 23$, and indirectly also IL-17) on the increase of risk of cardiovascular episodes, which affected the FDA decision about not approving the drug. No similar adverse reactions were observed after treatment with other anti-IL-12/23 and anti-IL-17 preparations [30]. The study conducted by us did not show a correlation of the levels of the analyzed cytokines with arterial hypertension in patients with psoriasis.

\section{Conclusions}

Biopharmaceuticals are the future for the treatment of many conditions with unconfirmed pathogenesis, including psoriasis. Genome studies are being conducted with a view to evaluating the potential response and the time to develop resistance to biopharmaceuticals. It also seems very important to study immunologic mechanisms responsible for the presence and severity of psoriasis, in order to personalize the therapy in the future and optimize the effect of action on the basic disease and on concomitant disorders, e.g. anti-IL-17 for patients with psoriasis with concomitant dyslipidemia and diabetes. Further detailed studies on the levels of cytokines in patients with psoriasis and metabolic syndrome are necessary.

\section{Conflict of interest}

The authors declare no conflict of interest.

\section{References}

1. Parisi R, Symmons DP, Griffiths CE, et al. Global epidemiology of psoriasis: a systematic review of incidence and prevalence. J Invest Dermatol 2013; 133: 377-85.

2. Davidovici BB, Sattar N, Prinz J. Psoriasis and systemic inflammatory diseases: potential mechanistic links between skin disease and co-morbid conditions. I Invest Dermatol 2010; 130: 1785-96.

3. Liu Y, Krueger JG, Bowcock AM. Psoriasis: genetic associations and immune system changes. Genes Immun 2007; 8: 1-12.

4. Arican O, Aral M, Sasmaz S, Ciragil P. Serum levels of TNFalpha, IFN-gamma, IL-6, IL-8, IL-12, IL-17, and IL-18 in patients with active psoriasis and correlation with disease severity. Mediators Inflamm 2005; 2005: 273-9.

5. Ni C, Chiu MW. Psoriasis and comorbidities: links and risks. Clin Cosmet Investig Dermatol 2014; 7: 119-32.

6. Fisman EZ, Motro M, Tenenbaum A. Cardiovascular diabetology in the core of a novel interleukins classification: the bad, the good and the aloof. Cardiovasc Diabetol 2003; 2: 11.

7. Voiculescu VM, Lupu M, Papagheorghe L, et al. Psoriasis and metabolic syndrome: scientific evidence and therapeutic implications. J Med Life 2014; 7: 468-71.

8. Danielsen K, Wilsgaard T, Olsen AO, et al. Elevated odds of metabolic syndrome in psoriasis: a population-based study of age and sex differences. Br J Dermatol 2015; 172: 419-27.

9. Langan SM, Seminara NM, Shin DB, et al. Prevalence of metabolic syndrome in patients with psoriasis: a populationbased study in the United Kingdom. I Invest Dermatol 2012; 132: 556-62.

10. Azfar RS, Seminara NM, Shin DB, et al. Increased risk of diabetes mellitus and likelihood of receiving diabetes mellitus treatment in patients with psoriasis. Arch Dermatol 2012; 148: 995-1000.

11. Armstrong EJ, Harskamp CT, Armstrong AW. Psoriasis and major adverse cardiovascular events: a systematic review and meta-analysis of observational studies. J Am Heart Assoc 2013; 2: e000062.

12. Micha R, Imamura F, Wyler von Ballmoos M, et al. Systematic review and meta-analysis of methotrexate use and risk of cardiovascular disease. Am J Cardiol 2011; 108: 1362-70.

13. De Vecchis R, Baldi C, Palmisani L. Protective effects of methotrexate against ischemic cardiovascular disorders in patients treated for rheumatoid arthritis or psoriasis: novel therapeutic insights coming from a meta-analysis of the literature data. Anatol J Cardiol 2016; 16: 2-9.

14. Marks JL, Edwards CJ. Protective effect of methotrexate in patients with rheumatoid arthritis and cardiovascular comorbidity. Ther Adv Musculoskelet Dis 2012; 4: 149-57.

15. Hugh J, Van Voorhees AS, Nijhawan RI, et al. From the Medical Board of the National Psoriasis Foundation: The risk of cardiovascular disease in individuals with psoriasis and the potential impact of current therapies. J Am Acad Dermatol 2014; 70: 168-77. 
16. Roubille C, Richer $\mathrm{V}$, Starnino T, et al. The effects of tumour necrosis factor inhibitors, methotrexate, non-steroidal antiinflammatory drugs and corticosteroids on cardiovascular events in rheumatoid arthritis, psoriasis and psoriatic arthritis: a systematic review and meta-analysis. Ann Rheum Dis 2015; 74: 480-9.

17. Ucak S, Ekmekci TR, Basat O, et al. Comparison of various insulin sensitivity indices in psoriatic patients and their relationship with type of psoriasis. I Eur Acad Dermatol Venereol 2006; 20: 517-22.

18. Boehncke S, Thaci D, Beschmann H, et al. Psoriasis patients show signs of insulin resistance. Br J Dermatol 2007; 157: 1249-51.

19. Fantuzzi G. Adipose tissue, adipokines, and inflammation. J Allergy Clin Immunol 2005; 115: 911-9.

20. Wellen KE, Hotamisligil GS. Inflammation, stress, and diabetes. J Clin Invest 2005; 115: 1111-9.

21. Solomon DH, Massarotti E, Garg R, et al. Association between disease-modifying antirheumatic drugs and diabetes risk in patients with rheumatoid arthritis and psoriasis. JAMA 2011; 305: 2525-31.

22. Pina T, Armesto S, Lopez-Mejias R, et al. Anti-TNF-alpha therapy improves insulin sensitivity in non-diabetic patients with psoriasis: a 6-month prospective study. J Eur Acad Dermatol Venereol 2014; 29: 1325-30.

23. Wu JJ, Liu L, Asgari MM, et al. Initiation of TNF inhibitor therapy and change in physiologic measures in psoriasis. J Eur Acad Dermatol Venereol 2014; 28: 1380-7.

24. Famenini S, Sako EY, Wu JJ. Effect of treating psoriasis on cardiovascular co-morbidities: focus on TNF inhibitors. Am J Clin Dermatol 2014; 15: 45-50.

25. Eirís N, González-Lara L, Santos-Juanes J, et al. Genetic variation at IL12B, IL23R and IL23A is associated with psoriasis severity, psoriatic arthritis and type 2 diabetes mellitus. J Dermatol Sci 2014; 75: 167-72.

26. Bellemore SM, Nikoopour E, Krougly O, et al. Pathogenic T helper type 17 cells contribute to type 1 diabetes independently of interleukin-22. Clin Exp Immunol 2016; 183: 380-8.

27. Marwaha AK, Tan S, Dutz JP. Targeting the IL-17/IFN-gamma axis as a potential new clinical therapy for type 1 diabetes. Clin Immunol 2014; 154: 84-9.

28. Roohi A, Tabrizi M, Abbasi F, et al. Serum IL-17, IL-23, and TGF-beta levels in type 1 and type 2 diabetic patients and age-matched healthy controls. Biomed Res Int 2014; 2014: 718946.

29. Ma C, Harskamp CT, Armstrong EJ, Armstrong AW. The association between psoriasis and dyslipidaemia: a systematic review. Br J Dermatol 2013; 168: 486-95.

30. Owczarczyk-Saczonek A, Placek W. Interleukin-17 as a factor linking the pathogenesis of psoriasis with metabolic disorders. Int J Dermatol 2017; 56: 260-8.

31. Varshney P, Narasimhan A, Mittal S, et al. Transcriptome profiling unveils the role of cholesterol in IL-17A signaling in psoriasis. Sci Rep 2016; 6: 19295.

32. Madhur MS, Lob HE, McCann LA, et al. Interleukin 17 promotes angiotensin II-induced hypertension and vascular dysfunction. Hypertension 2010; 55: 500-7. 